\title{
Sexual selection rewires reproductive protein networks
}

4 Timothy L. Karr ${ }^{1}{ }^{\star} \S$, Helen Southern², Matthew Rosenow ${ }^{3}$, Toni I. Gossmann², and Rhonda R.

5 Snook $^{4, *}$

6

7 'Department of Genomics and Genetic Resources, Kyoto Institute of Technology, Kyoto,

8 Japan

$9 \quad{ }^{2}$ Department of Animal and Plant Sciences, University of Sheffield, UK.

$10 \quad{ }^{3}$ Caris Life Sciences, Phoenix, AZ

$11{ }^{4}$ Department of Zoology, Stockholm University, Sweden

12 *Corresponding authors: Email: tkarr@asu.edu \& rhonda.snook@zoologi.su.se

13 §Current address: Arizona State University, School of Life Sciences, Tempe, AZ 85287

16 Running title: Sexual selection and protein interaction networks

17 The abbreviations used are:

18 BLAST, Basic Local Alignment Search Tool

19 Dpse, Drosophila pseudoobscura

20 PCSS, postcopulatory sexual selection

21 SFPs, seminal fluid proteins

22 Dmel, D. melanogaster

23 SDS, sodium dodecylsulfate 
24 SDS-PAGE, sodium dodecylsulfate polyacrylamide gel electrophoresis

25 MS, mass spectrometry

26 LC-MS/MS, liquid chromatography-MS/MS

27 AcgP, accessory gland proteome

28 FDRs, False Discovery Rates

29 AcgS, accessory gland secretome

30 exoP, exoproteome

31 LFQ, label-free quantitation

32 P, polyandry

$33 \mathrm{M}$, monandry

34 GO, gene ontology

35 CC, cellular component

36 MF, molecular function

37 BP, biological process

38 STRING, Search Tool for the Retrieval of Interacting Genes/Proteins

39 DIOPT, DRSC Integrative Ortholog Prediction Tools

$40 \quad E R$, endoplasmic reticulum 
41 Polyandry drives postcopulatory sexual selection (PCSS), resulting in rapid evolution

42 of male ejaculate traits. Critical to male and female fitness, the ejaculate is known to 43 contain rapidly evolving seminal fluid proteins (SFPs) produced by specialized male secretory accessory glands. The evidence that rapid evolution of some SFPs is driven by PCSS, however, is indirect, based on either plastic responses to changes in the sexual selection environment or correlative macroevolutionary patterns. Moreover, such studies focus on SFPs that represent but a small component of the accessory gland proteome. Neither how SFPs function with other reproductive proteins, nor how PCSS influences the underlying secretory tissue adaptations and content of the accessory gland, has been addressed at the level of the proteome. Here we directly test the hypothesis that PCSS results in rapid evolution of the entire male accessory gland 52 proteome and protein networks by taking a system-level approach, combining 53 divergent experimental evolution of PCSS in Drosophila pseudoobscura (Dpse), high 54 resolution mass spectrometry (MS) and proteomic discovery, bioinformatics and population genetic analyses. We demonstrate that PCSS influences the abundance of over 200 accessory gland proteins, including SFPs. A small but significant number of these proteins display molecular signatures of positive selection. Divergent PCSS also results in fundamental and remarkably compartmentalized evolution of accessory gland protein networks in which males subjected to strong PCSS invest in protein networks that serve to increase protein production whereas males subjected to relaxed

$61 \quad$ PCSS alters protein networks involved in protein surveillance and quality. These results 62 directly demonstrate that PCSS is a key evolutionary driver that shapes not only 63 individual reproductive proteins, but rewires entire reproductive protein networks. 
64 Polyandry, in which females mate with different males across a reproductive bout, generates

65 PCSS in which ejaculates compete for fertilization of a limited supply of ova and females

66 may choose whose sperm will fertilize those limited ova (1). Polyandy also engenders sexual

67 conflict, in which male and female reproductive interests differ, as a consequence of the

68 disproportionate costs and benefits of mating between the sexes (2). PCSS operates in

69 internally fertilizing organisms between the female reproductive tract and the male ejaculate,

70 which is composed of both sperm and non-sperm components, including SFPs (3). SFPs

71 were first identified by their canonical signal peptide sequence that direct proteins to the secretory pathway (4). Cross-species comparative work has found that general classes of SFPs are conserved (e.g., proteases and protease inhibitors, lectins and prohormones) suggesting that their mechanisms of action are also conserved. However, individual SFPs can rapidly evolve with signals of accelerated rates of adaptive molecular evolution found in studies of coding sequence and male-biased gene expression observed across different animal taxa (e.g., mammals (5, 6); birds (7); Drosophila (8-10)).

Rapid evolution of SFPs is attributed to PCSS because this cocktail of proteins, transferred to 80 females, has dramatic and sometimes antagonistic effects on male and female fitness, 81 including increased female fecundity, reduced female receptivity, decreased female life span, 82 and remodelling of female reproductive tract morphology (4, 11-13). Moreover, males can 83 tailor ejaculate composition, altering the quantity of specific SFPs, via plastic changes in 84 response to variation in the PCSS environment $(5,14)$ although a mechanism by which males 85 accomplish this is unknown. However, while many studies have examined the evolution and 86 fitness consequences of SFPs, most of them examine the impact of one or just a few of these 87 proteins. This focus is problematic because SFPs do not work in isolation, but rather as partners in protein-protein and protein-nucleic acid networks, and not just solely with other SFPs $(15,16)$. Therefore, changes in either protein levels or protein function may have 90 significant up- and/or down-stream consequences on organismal physiology and fitness. 
92 other proteins also produced by male accessory gland tissue that may interact with SFPs and

93 influence fitness.

94

95 Most of our current knowledge of SFPs comes from traditional protein detection methods and 96 comparisons aimed at one species, D. melanogaster $(9,17-19)$. However, the advent of 97 sophisticated transcriptomic, proteomic and bioinformatics approaches have identified new 98 SFPs and associated reproductive proteins not only in Dmel but also other taxa (20-26).

99 Furthermore, high throughput mass spectrometry based techniques now routinely produce 100 cellular and tissue proteomes consisting of thousands of protein IDs not only useful for protein 101 identification but also the analysis and identification of protein interaction networks.

103 Initial application using these techniques to the analysis of the sperm proteome $(24,25)$ found 104 heterogeneity in the evolutionary rates of genes compared to SFPs which lead to the 105 hypothesis that adaptive compartmentalization can occur both within and between proteomes 106 involved in reproduction. This hypothesis suggests that interacting reproductive proteins would 107 comprise a set of "core" proteins with essential cellular functions (e.g., sperm motility) that are 108 predicted to be under strong purifying selection and another set of proteins under adaptive 109 selection generated from PCSS, e.g., sperm-egg interactions (24). Other than sperm, direct 110 empirical confirmation of adaptive compartmentalization, and a possible role of PCSS in this 111 process, has yet to be undertaken. Likewise, application of high throughput mass spectrometry 112 based techniques have allowed characterization and dynamics of protein interaction networks 113 in a variety of cellular contexts including disease-induced perturbation and or over evolutionary 114 time frames (see $(27,28)$ for recent reviews). However, the role of PCSS in shaping the 115 architecture of protein interaction networks is unknown.

117 Here we address the overall qualitative and quantitative impact of PCSS on the male 118 accessory gland proteome and directly test the hypothesis that PCSS effects SFP production 119 and their reproductive protein networks. We also test for signatures of positive selection and 
120 examine evidence for adaptive compartmentalization. We perform these tests using replicated

121 experimentally evolved lines of $D$. pseudoobscura (Dpse) in which PCSS was manipulated

122 over many generations by either allowing polyandry ("P" lines; 1 female with 6 males) or

123 enforcing monogamy ("M" lines; 1 female with 1 male) (29). Previous studies using these lines

124 have found that divergent sexual selection impacts the evolution of reproductive traits relevant

125 to PCSS and SFP production, including P males altering female investment in both early and

126 total progeny production (30) and P males having larger accessory glands that resulted in

127 greater reproductive success (31). Sex-biased gene expression has also evolved in these

128 lines $(32,33)$, and, taken togther these results strongly motivate using these lines to directly

129 test the role of PCSS in shaping male reproductive proteomes at the microevolutionary scale.

\section{EXPERIMENTAL PROCEDURES}

132 Experimental lines. The establishment and maintenance of the selection lines were previously 133 described in detail (29) Briefly, an ancestral wild-caught population of Dpse from Tucson 134 Arizona USA, a naturally polyandrous species (wild caught females have been shown to be 135 frequently inseminated by at least two males at any given time; (34)), was used to establish 136 the selection lines. From this population, four replicate lines (replicates 1-4) of two different 137 sexual selection treatments were established. To modify the opportunity for sexual selection, 138 adult sex ratio in vials was manipulated by either confining one female with a single male and 139 enforcing monogamy ("monogamy" treatment, M) or one female with six males promoting 140 polyandry ("polyandry" treatment, P (NB: this treatment has also been referred to as E in other 141 publications). Effective population sizes are equalized between the treatments as described 142 previously (35). At each generation, offspring are collected and pooled together for each 143 replicate line, and a random sample from this pool is used to constitute the next generation in 144 the appropriate sex ratios, thus proportionally reflecting the differential offspring production 145 across families. In total, eight selection lines (M1, M2, M3, M4 and P1, P2, P3, P4) are 146 maintained, in standard vials $(2.5 \times 80 \mathrm{~mm})$, with a generation time of 28 days. All populations 147 are kept at $22^{\circ} \mathrm{C}$ on a $12 \mathrm{~L}: 12 \mathrm{D}$ cycle, with standard food media and added live yeast. 
149 Experimental flies. Flies from replicates 1-4 of each of the selection lines were collected from

150 generations 157, 156, 155 and 153 respectively. We standardized for maternal and larval

151 environments (29), but in brief, parental flies were collected and housed en-mass in food

152 bottles, then groups of about 30 were transferred on egg laying plates for 24 hours, removed

153 and replaced with a fresh egg plate. This second plate was removed after 24 hours, then 48

154 hours later, first instar larvae were collected in groups of 100 and housed in standard

155 molasses/agar food vials at $22^{\circ} \mathrm{C}$. Males from these vials were collected on the day of eclosion

156 and housed in vials of 10 individuals, until they were sexually mature (36), and then dissected

157 when they were 5 or 6 days old.

159 Accessory gland tissue preparation. For dissections, males were anaesthetized with ether, 160 placed in a drop of phosphate buffered saline and the reproductive tract removed. Intact 161 accessory glands were clipped from the rest of the reproductive tract (Supplemental Figure $1621 \mathrm{~A}, \mathrm{~B})$ with fine dissection needles, moved to another drop of phosphate buffered saline for 163 rinsing, and then transferred to a microcentrifuge tube. 30 accessory glands per replicate were 164 used for subsequent LC-MS/MS as described below. Samples from each replicate were 165 solubilized at $4^{\circ} \mathrm{C}$ by addition of $30 \mu$ of RIPA buffer (Sigma), and a HALT protease inhibitor 166 mixture containing phenylmethylsulfonyl fluoride (Thermo Fisher Scientific). Once all 167 accessory glands had been dissected, samples were freeze/thawed three times on dry ice $(\sim 5$ $168 \mathrm{mins}$ ) and then thawed at $37^{\circ} \mathrm{C}$ for 30 seconds. After the freeze/thaw cycles, samples were 169 vortexed for 30 seconds, centrifuged at $20,000 \mathrm{rpm}$ for 5 minutes at $4^{\circ} \mathrm{C}$ and then stored at $17080^{\circ} \mathrm{C}$ until processing.

172 Sodium dodecylsulfate polyacrylamide gel electrophoresis (SDS-PAGE) and in-gel digestion 173 of proteins. Protein concentration was determined using a Bradford assay and samples were 174 solubilized in SDS sample buffer containing $10 \mathrm{mM}$ dithiothreitol and proteins separated on 4$17512 \%$ SDS-PAGE gels per manufacturer instructions (Invitrogen). Protein bands were 
176 visualized (Supplemental Figure 1C) using Brilliant Blue G Colloidal Concentrate (Sigma).

177 Each gel lane was manually cut into approximately equivalent sized pieces and destained

178 using $200 \mathrm{mM}$ ammonium bicarbonate and $40 \%$ acetonitrile. Gel pieces were then reduced in

$179200 \mu \mathrm{l}$ of a $50 \mathrm{mM}$ ammonium bicarbonate buffer containing $10 \mathrm{mM}$ dithiothreitol, followed by

180 alkylation in a similar volume of a $50 \mathrm{mM}$ ammonium bicarbonate containing $55 \mathrm{mM}$

181 iodoacetamide. Gel pieces were then centrifuged at $13 \mathrm{Kg}$ for 10 seconds and dried using a

182 vacuum concentrator until all samples were dry ( 30 $\mathrm{min})$. The dried pieces were then

183 hydrated in a solution containing $20 \mu \mathrm{l}$ of trypsin (New England BioLabs) and $50 \mu \mathrm{l}$ of

184 acetonitrile and incubated overnight at $37^{\circ} \mathrm{C}$. Peptides were extracted the following day using

185 a standard method with a solution of $100 \%$ acetonitrile and $5 \%$ formic acid and dried down

186 overnight in a vacuum concentrator at $30^{\circ} \mathrm{C}$. Resulting peptides were resuspended in $7.5 \mu \mathrm{l}$

187 of $0.1 \%(\mathrm{v} / \mathrm{v})$ formic acid, $3 \%(\mathrm{v} / \mathrm{v})$ acetonitrile, sonicated in a water bath for 5 minutes and

188 centrifuged at $13 \times \mathrm{g}$ for 10 seconds, before being transferred to a sample vial and loaded into

189 the autosampler tray of the Dionex Ultimate $3000 \mu$ HPLC system. Samples were set to run

190 using the Xcalibur sequence system.

192 Liquid chromatography-MS/MS (LC-MS/MS) data collection. All MS data were collected using

193 an LTQ Orbitrap Elite hybrid mass spectrometer (Thermo Fisher Scientific) equipped with an

194 Easy-Spray (Thermo Fisher Scientific) ion source. Peptides were separated using an Ultimate

1953000 Nano LC System (Dionex). Peptides were desalted on-line using a capillary trap column

196 (Acclaim Pepmap100, $100 \mu \mathrm{m}, 75 \mu \mathrm{m} \times 2 \mathrm{~cm}, \mathrm{C} 18,5 \mu \mathrm{m}$; Thermo Fisher Scientific) and then

197 separated using 60 min reverse phase gradient (3-40\% acetonitrile/0.1\% formic acid) on an

198 Acclaim PepMap100 RSLC C18 analytical column $(2 \mu \mathrm{m}, 75 \mu \mathrm{m}$ id x 10 cm; Thermo Fisher

199 Scientific) with a flow rate of $0.25 \mu \mathrm{l} / \mathrm{min}$. The mass spectrometer was operated in standard

200 data dependent acquisition mode controlled by Xcalibur 2.2. The instrument was operated

201 with a cycle of one MS (in the Orbitrap) acquired at a resolution of 60,000 at $\mathrm{m} / \mathrm{z} 400$, with the

202 top 20 most abundant multiply-charged (2+ and higher) ions in a given chromatographic

203 window further subjected to CID fragmentation in the linear ion trap. An FTMS target value of 
$2041 \mathrm{e} 6$ and an ion trap MSn target value of 10000 were used. Dynamic exclusion was enabled

205 with a repeat duration of $30 \mathrm{~s}$ with an exclusion list of 500 and exclusion duration of $30 \mathrm{~s}$.

206 Experimental Design and Statistical Rationale

207 Database construction of the Dpse accessory gland proteome (AcgP). The mass spectra data

208 files were searched individually using Sequest HT within the Proteome Discover suite (Thermo

209 Fisher Scientific, San Jose, CA, USA; version 1.4.1.14) using Drosophila pseudoobscura

210 pseudoobscura fasta file, (December, 2015 release). Peptide matches were further analyzed

211 and validated within Scaffold Q+ (Proteome Software; version 3.2.0) using X!Tandem.

212 Sequest HT and X!Tandem searches were set with a fragment ion mass tolerance of 0.60 Da

213 and a parent ion tolerance of 10.0 parts per million. The oxidation of methionine (15.99),

214 carboxyamidomethyl of cysteine (57.02), and acetyl modification on peptide $\mathrm{N}$-terminus

215 (42.01) were set as variable modifications. Files from Sequest HT searches within the same

216 gel lane were merged together as Mudpit using Scaffold which calculated False Discovery

217 Rates (FDRs) using a reverse concatenated decoy database (FDR was set at 1.0\%). Peptide

218 identifications were accepted if they could be established at greater than $95.0 \%$ probability as

219 specified by the PeptideProphet ${ }^{48}$ and protein identifications were accepted if they could be

220 established at greater than $99.0 \%$ probability and contained at least 2 identified peptides.

221 Protein probabilities were assigned by the Protein Prophet Algorithm (37). Proteins that

222 contained similar peptides and could not be differentiated based on MS/MS analysis alone

223 were grouped to satisfy the principles of parsimony. The dataset was filtered so that every

224 protein must be identified by at least two unique peptides in any one of the biological replicates.

225 Although a conservative approach, this procedure ensured a robust dataset devoid of potential

226 misidentifications often caused by use of a single peptide for protein identification. To establish

227 a working list of the AcgP, protein IDs from Scaffold were converted to Dpse Fly Base gene

228 numbers (FBgns) using the Uniprot website (Uniprot.org). The resulting Dpse FBgns were

229 then used to query Flybase (Flybase.org) to retrieve orthologous Dmel genes from the

230 OrthoDB orthology tables as implemented in Flybase (38). A complete listing can be found in

231 Supplemental Table 1. 
233 Database construction of the Dpse accessory gland secretome (AcgS) and exoproteome

234 (exoP). As a secretory organ, the accessory gland is expected to contain the cellular

235 machinery necessary for efficient and sustained secretory activity throughout the adult

236 reproductive life cycle. To examine and focus on potential activities related to secretion, we

237 assembled an in silico AcgS from the $3281 \mathrm{FBgns}$ of the AcgP as input into Uniprot resulting

238 in 5624 UniProtKB IDs (which includes all predicted protein isoforms). Fasta protein sequence

239 files from each Uniprot entry were downloaded and input into SignalP

240 (http://www.cbs.dtu.dk/services/SignalP/) (39) and Phobius (http://phobius.sbc.su.se) (40),

241 using default settings. The protein IDs were combined, exported into Excel, yielding a final list

242 of 771 UniProt identifiers. The Uniprot IDs were mapped back to 535 unique Dpse FBgns (via

243 Uniprot) which, after submission to OrthoDB (via Flybase) resulted in a final list and 506 Dmel

244 orthologs. Candidate SFPs were identified by first querying the list of 515 AcgS genes in

245 Flybase for Gene Ontology (GO) terms containing "extracellular". The resulting list of 151

246 proteins therefore represents the accessory gland exoproteome (exoP) and is considered to

247 contain a representative sampling of a major fraction of SFPs.

248

249 Network and protein interaction network analyses. The finalized datasets were used for

250 downstream bioinformatic analyses and subsequent visualizations of $\mathrm{GO}$ enrichment. The

251 protein coding sequences of the AcgP were downloaded from Uniprot and submitted to

252 Blast2go for annotation and tabulation of the three major GO categories, biological process

253 (BP), molecular function (MF) and cellular component (CC). GO enrichment and network

254 visualization and analysis was performed with Cytoscape v3.4 $(41,42)$ and ClueGO plugin

255 version 2.2.4 $(43,44)$. Network parameters used were specific for each dataset as detailed in

256 figure legends and supplemental tables. Protein interaction network analysis was performed

257 using Search Tool for the Retrieval of Interacting Genes/Proteins (STRING), a program that

258 calculates the degree of protein-protein network interconnectivity (45). A combined set of 
259 differentially abundant proteins from each $\mathrm{M}$ - and P-line (Qspec, see next section below) was

260 uploaded to the STRING website and the analysis run using the "high confidence 0.9" setting

261 and the "experimental" and "databases" selected for "evidence". Proteins in each M- and P-

262 up groups were distinguished by color-code: red $(M>P)$ and green $(P>M)$.

263

264 Label-free quantitation and statistical analysis. To test for differential abundance of male

265 reproductive proteins in response to postcopulatory sexual selection statistical signifcance

266 was calculated using a Bayesian approach as implemented by Qspec (46), part of the

267 statistical package Qprot (47). Qspec provides a statistics framework that calculates Bayes

268 factors- essentially likelihood measures of significance in the context of a generalized linear

269 mixed effects model. Local implementation of Qprot (http://sourceforge.net/p/qprot/) provided

270 command line access to QSpec that calculated Z-statistics, log-fold change estimates and a

271 local FDR for each pair-wise comparison. This approach has been shown effective in capturing

272 a broad range of differentially abundant proteins using LFQ methods $(46,48)$. Raw spectral

273 counts of the $M$ and $P$ line datasets from Scaffold were input into Qspec and the output

274 imported into Excel. Differential protein abundance differences were calculated using a $1 \%$

275 false-discovery rate (FDR) cut-off, and proteins assigned based on log-fold changes relative

276 to the $M$-line, i.e., positive fold-change, $M<P$; negative fold-change, $M>P$.

277

278 Molecular evolution parameters. We obtained coding sequence information for the genomes

279 of the two close relatives of Dpse, D. lowei and D. affinis, downloaded from

280 http://popoolation.at/lowei genome and http://popoolation.at/affinis genome (49). These two

281 species have the same karyotype as Dpse and show reasonable divergence (median pairwise

$282 \mathrm{dS}=0.102$ versus $D$. lowei and $d S=0.26$ versus $D$. affinis) thus avoiding substitution rate

283 saturation. To identify orthologs of the identified AcgP Dpse proteins in the two other

284 Drosophila genomes we combined two approaches. First we used gene annotation ignoring

285 isoforms specification (as these are difficult to identify within a proteomic screen). We then 
286 used best BLAST hits (50) of the Dpse gene against each of the two other genomes, but 287 excluded gene sets for which annotation was contradictory to the Dpse annotation. Using a 288 pipeline we developed earlier (51), sequences were aligned using MUSCLE (52), uncertain sequences filtered out using ZORRO (53) and input files converted with pal2nal (54). Sequences were then analyzed using PAML v4.9 (55) to obtain dN/dS values for each gene set (one-ratio estimates) as well as to conduct direct tests of positive selection as implemented 292 in the site-specific models models M7a and M8a. For the one-ratio estimates, median 293 differences in dN, dS and dN/dS among groups (AcgP, AcgS, exoP and differentially abundant $294 \mathrm{M}$ and $\mathrm{P}$ proteins) were tested using a non-parametric two-tailed test (57). For the site tests of positive selection, we obtained significance by conducting LRTs as described in the PAML 296 manual and corrected P-values for multiple testing using an FDR=0.1 using the method of

297 Benjamini and Hochberg (56). Median differences in dN, dS and dN/dS among groups (AcgP, 298 AcgS, ExoP and differentially abundant $\mathrm{M}$ and $\mathrm{P}$ proteins) were tested using a non-parametric 299 two-tailed test (57). Enrichment between counts per groups were tested using a 2x2 Fisher's 300 exact test.

\section{RESULTS}

304 The Dpse AcgP. A Dpse AcgP was constructed from peptide-based ("bottom-up") shotgun MS/MS spectral data obtained from eight independent runs consisting of four replicate samples from each of two Dpse experimental sexual selection populations. For the purposes

307 of assembling a proteome with the broadest coverage, data from all runs were pooled together 308 resulting in a total of 3757 UniProt IDs that mapped to 3281 unique FlyBase gene names. The 309 M- and P- datasets were highly correlated with $>90 \%(3534 / 3757)$ overlap (Figure 1A). 310 Likewise, proteins with values in all four replicates for each PCSS treatment represented the 311 majority of identified proteins (M-line 2103/3649; 57.6\% and P-line, 2235/3642; 61.4\%) A 312 complete listing and tabulation of these results can be found in Supplemental Table S1. The 313 small number of proteins unique to each population ( $\mathrm{M}$-unique = 115; $\mathrm{P}$-unique = 108; Figure 
314 1A) most likely represent missed protein assignments due to low quantities (as measured by

315 total spectral counts). Indeed, the average total spectral counts for the unique set of proteins

316 (ave. $4.5 ; n=223$ ) was 16-fold lower than the average across the entire dataset (ave. $72.8, n$

$317=3874)$.

318

319 GO and pathway analysis of the AcgP

320 Pathway and functional analyses began by identifying orthologous Dmel genes, (3159/3281,

$32196.2 \%$; Supplemental Table 1), providing a useful annotated database to evaluate the overall

322 patterns of the functional elements in the AcgP. As an overview of the major GO groupings,

323 Blast2Go returned "signaling", "reproduction" and "localization" among BP- and numerous

324 proteins annotated as extracellular involved in the CC-categories, respectively (Supplemental

325 Figure 2). Statistical analysis for GO category enrichment (Figure 2) identified BP terms

326 related to intracellular transport $(n=363, P=2.80 E-45)$, translation $(n=257, P=2.47 E-35)$,

327 establishment of protein localization $(n=418, P=1.22 E-44)$, vesicle-mediated transport $(n=$

$328301, P=8.47 E-42)$, endocytosis $(n=106, P=7 E-10)$, and secretion $(n=140, P=2.71 E-16)$.

329 Likewise, the AcgP contains a sgnificant number of proteins in CC categories annotated as

330 "extracellular region" ( $n=266, P=3.2 E-05)$, "endomembrane system" $(n=583, P=1.04 E-$

$33157)$ and "vesicle" ( $n=230, P=8.72 E-25)$. Finally, the overall known biochemical pathways of

332 the AcgP, analyzed using the Kyoto Encyclopedia of Genes and Genomes (KEGG), revealed

333 a similar enrichment of 20 overview terms curated by KEGG that included ribosome

334 biogenesis, protein export, endocytosis and the Wnt signaling pathways (Table 1). We

335 conclude the AcgP contains features expected of a metabolically active cell enriched for

336 protein production and protein secretion. See Supplemental Table 2 for a complete listing of

337 all enriched GO categories.

339 The Dpse accessory gland secretome (AcgS). Signal peptides are a ubiquitous class of short

340 (20-22 aa) N-terminal sequences, that target proteins for translocation across, and into, the 341 endomembrane system of the cell $(58,59)$. Collectively proteins containing signal peptide 
342 sequences are considered part of the secretory pathway, and a subclass - those secreted into

343 the extracellular space - are termed the secretome (also referred to as the exoproteome,

344 exoP). Therefore, some or all components of the exoP can be considered as candidate SFPs.

345 Given the secretory nature of the Drosophila accessory gland, we therefore queried the AcgP

346 for proteins containing canonical signal sequences using two predictive programs, SignalP

347 and Phobius (see Methods). SignalP (39) is a neural network-based algorithm designed to

348 detect canonical $\mathrm{N}$-terminal signal sequences and discriminate against $\mathrm{N}$-terminal

349 transmembrane regions known to reduce predictive power, and Phobius utilizes a combined

350 model of both transmembrane and signal peptide predictors $(40,60)$. The combined output of

351 both resulted in 771 Uniprot IDs that mapped to 535 Dpse FBgns (Figure 1B; Supplemental

352 Table 3). Dmel orthologs (OrthoDB via Flybase) subsequently returned 506 Dmel orthologs to

353 the Dpse AcgS including a small percentage $(8 / 511,1.6 \%)$ of "1:many" matches included in

354 the analysis to capture the greatest proteome coverage of the secretome. Thus, the AcgS

355 represents approximately $15 \%(535 / 3281 ; 16.3 \%)$ of the entire AcgP consistent with similar

356 calculations for the predicted human secretome $(\sim 15 \%$,

357 http://www.proteinatlas.org/humanproteome/secretome). Finally, the DRSC Integrative

358 Ortholog Prediction Tools (DIOPT) website (http://www.flyrnai.org/diopt) identified 61\%

$359(327 / 535)$ of the AcgS that corresponded to orthologous human sequences (Supplemental

360 Table 3).

361

362 Gene Ontology (GO) Functional Analysis of the AcgS

363 The high degree of homology between the Dpse AcgS genes and Dmel (506/528),

364 Supplemental Table 3) provided a putative orthologous secretome useful for GO analysis and

365 network visualization. A significant enrichment in BP terms was observed, many related to

366 multicellular organism reproduction $(n=113, p=7 E-7)$, reproduction $(n=116, p=7 E-7)$, behavior

$367(n=53, p=2.1 E-5$ and proteolysis $(n=66, p=3.5 E-6)$. The secretome is enriched in MF terms

368 related to oxidoreductase activity $(n=55, p=7.1 \mathrm{E}-7)$ and hydrolase activity $(n=138, p=8.5 e-14$;

369 see Supplemental Table 3 for a complete list of all AcgS enriched BP, CC and MF GO 
370 categories). We also examined the subcellular localization of the AcgS using the Cerebral

371 layout tool implemented in Cytoscape. As expected for functions related to secretion and

372 proteins containing signal sequences targeted to the secretory pathway, the predicted

373 subcellular localization of AcgS proteins were skewed toward extracellular and plasma

374 membrane proteins (Figure 3).

375

376 The AcgS contains a robust repertoire of putative SFPS

377 We used the AcgS to identify a list of 151 putative SFPs representing $>25 \%(151 / 506)$ of the

378 AcgS (Figure 1B; Supplemental Table 4). This list is a conservative estimate as 99 genes had

379 no CC functional annotation. Cytoscape and Cluego network analyses returned enriched BP

380 terms of major functional categories including, insemination, sperm competition, and

381 copulation reproduction, and negative regulation of endopeptidase activity (Table 2;

382 Supplemental Table 4). Compared with a list of 212 Dmel SFPs assembled from the literature

$383(9,20,21)$ an overlap of $32.1 \%(68 / 212)$ was observed that included 32 named SFP genes

384 including Acp53Ea, Acp53C14d and Acp53C14c (Supplemental Table 4).

385

386 Divergent sexual selection results in differential AcgP protein abundance

387 To examine the proteome-wide response to sexual selection, differential protein abundance

388 between the $\mathrm{M}$ - and P-lines was determined. A total of 250 differentially abundant Uniprot

389 entries were identified using LFQ and Qspec statistical analysis across both treatments (FDR

$390<1 \%)$. The Uniprot IDs were subsequently mapped to 229 orthologous Dmel proteins

391 (Supplemental Table 5). Taken together, network visualization of all 229 differentially

392 abundant proteins revealed tight clustering of GO terms grouped into processes related to the

393 cytoskeleton, protein translational machinery- particularly ribosomal proteins- and a suite of

394 amino-acyl tRNA synthetases, along with a significant enrichment in categories related to

395 endomembrane systems (e.g., Golgi, ER) and elements of the secretory pathway associated

396 with vesicles and the Golgi apparatus (Figure 4A). Moreover, $14.4 \%(33 / 229)$ of the

397 differentially abundant proteins were members of the exoP, including known Acps 
398 (Supplemental Table 6).

399

400

$\underline{M-\text { and } P \text {-line differential protein abundance }}$

401 To further probe the effect of PCSS on our divergent selection lines, we defined differential protein abundance between the sexual selection treatments based according to the sign of the log-fold change relative to the $M$-line (i.e., positive fold-change, $M<P$; negative foldchange, M > P; Supplemental Table 5). Of the 229 differentially abundant proteins, 133 and 96 were more abundant in the $\mathrm{M}$-line in the $\mathrm{P}$-line, respectively (Figure $1 \mathrm{C}$ ). To examine differences in the function and cellular location of proteins differentially abundant between the $\mathrm{M}$ - and P-lines, the overlap of the two with respect to their GO groupings was generated using Cytoscape and Cluego. This comparison revealed a striking separation of functional grouping, with each selection line showing unique sub-groupings within the overall network of functional annotations (Figure 4B). We found differentially abundant proteins related to translational machinery (e.g., ribosomes) and cytoskeleton groupings predominant within the P-line (Figure

4B, green) with bias toward cytoplasmic cellular activities whereas Golgi- and vesicle- related

413 groupings (including the exoP) biased in the M-line (Figure 4B, red) and functioning near or at 414 the cell periphery.

416 Thirty-three of the differentially abundant proteins (24 up in M, 9 up in P) were annotated as

417 "extracellular" (i.e., putative SFPs; Supplemental Table 6). While a larger number of candidate 418 SFPs were more abundant in M-line compared to $\mathrm{P}$-line males, over $50 \%$ of $\mathrm{P}$ abundant SFPs 419 overlapped with known Dmel SFPs (5/9) compared to $29 \%$ overlap between M abundant and 420 Dmel SFPs (7/24). Differentially abundant $\mathrm{P}$ male proteins did not show significant GO BP 421 enrichment whereas significant enrichment was found in M-line males related to "response to 422 fungus" ( $P=2.1 \mathrm{E}-02)$ and "wound healing" ( $P=2.0 \mathrm{E}-02)(9 / 24,34.5 \%$; Supplemental Table 6; Supplemental Figure 3). 
426 While the differentially abundant proteins of each selection line was biased towards distinct

427 sets of GO functional groupings (Figure 4B), we also tested whether these relationships

428 extended to specific protein interaction networks of known network topologies. We used the

429 most stringent filters for network interactions on STRING (45), and found a statistically

430 significant $(P=0.001) P P I$ network of 91 proteins containing nine defined subnetworks and

431 including elements of 34 KEGG pathways (Supplemental Table 7). A striking aspect of the

432 STRING-generated PPI networks was the almost complete segregation of $M$ and $P$

433 differentially abundant proteins into line-specific protein interaction networks (Figure 5). For

434 example, all elements of the "vesicle transport" and "purine biosynthesis" PPI networks were

435 more abundant exclusively in the M-line. Likewise, all proteins under "microtubule

436 organization" and almost all (15/18) in the "ribosome" category are more abundant in the P-

437 line. These results show that the divergent sexual selection treatments used to generate the

$438 \mathrm{M}$ - and P-lines resulted in highly focused and interrelated changes in protein abundance that

439 were specific to each treatment.

441 Molecular evolutionary rates of accessory gland protein genes

442 We tested for rates of molecular evolution in male reproductive proteins by estimating omega

443 (dN/dS substitution rates) for each set of proteins: candidate SFPs, secretome proteins

444 (minus SFPs), and the remaining accessory gland proteome proteins using orthologs from two

445 closely related species in the obscura group, $D$. lowei and $D$. affinis. We found that putative

446 Dpse SFPs (Exoproteome) are evolving faster than both accessory gland proteome proteins

447 (AcgP; median omega $=0.088$ versus $0.052, \mathrm{P}=\mathrm{E}-09)$ and accessory gland secretome minus

448 the Exoproteome (AcgS; median omega $=0.088$ versus $0.078, \mathrm{P}=2.7 \mathrm{E}-02$; Figure $6 \mathrm{~A})$. AcgS

449 proteins also evolve faster than the AcgP proteins (median omega $=0.052$ versus $0.078, P=$

$4501 \times 10-9 ;$ Figure 6A).

452 Given that sexual selection is hypothesized to result in rapid protein sequence evolution, we 453 then compared the evolutionary rates of the selection-specific differentially abundant proteins 
454 and accessory gland proteins (excluding selection-specific proteins). We found that the $\mathrm{P}$

455 treatment showed pronounced reduction in $\mathrm{dN}$ compared to the $\mathrm{M}$ treatment (Figure 6B,

$456 \mathrm{dN}=0.009(\mathrm{P})$ versus $0.018(\mathrm{M}), \mathrm{P}=0.011)$. This is perhaps unsurprising considering that

457 cytosolic ribosomal proteins are highly enriched in $\mathrm{P}$, a protein group that has been shown to

458 be slowly evolving (61). However, slowly evolving genes may be indicative of intensified

459 selective pressures which obscure signals of positive selection, so to account for this we

460 employed a direct site-specific test of positive selection across the accessory gland proteome.

461 We identified 19 proteins that showed positive selection, 14 from the AcgP and 5 showing

462 differential abundance in the selection lines, 3 in $P$ and 2 in $M$ (Table 3). An enrichment

463 analysis shows that genes encoding proteins that were differentially abundant following

464 experimental microevolution were more likely to be under positive selection than those in the

465 AcgP set (Figure 6C, Supplemental Table 8) after correction for multiple testing and this is

466 significant for the P-only set as well.

467

468 DISCUSSION

469 Proteomics of the Dpse male accessory gland

470 The main aim of this work was to directly test the role of postcopulatory sexual selection in the

471 evolution of reproductive proteins and the male accessory gland reproductive protein network.

472 To accomplish this aim, we employed a "bottom up" shotgun proteomics approach, generating

473 a robust accessory gland proteome containing 3281 proteins, representing the first accessory

474 gland proteome to be described in Drosophila. The AcgP proteins in Dpse were enriched for

475 cellular components expected from a tissue whose primary function is secretory, including

476 several cellular component GO terms related to membranes, extracellular regions, and

477 peptidase complex. The top biological process GO terms clearly indicated a large investment

478 in processes directly and indirectly related to protein synthesis, protein assembly, transport

479 and secretion. We then in silico concatenated this list to include proteins with secretory signal

480 sequences to identify 545 accessory gland secretome proteins, which were enriched for GO 
481 terms related to the biological processes of reproduction, behavior and proteolysis, with these

482 proteins heavily biased towards subcellular localizations in the plasma member or extracellular 483 components, as predicted from proteins with secretory signals.

\section{Candidate Dpse SFPs contains many known Dmel SFPs}

486 SFPs are secreted from the accessory gland tissue and transferred to the females upon 487 mating. To generate a list of putative SFPs, we restricted the AcgS to only those proteins that 488 were associated with GO cellular component annotations containing the keyword 489 "extracellular". The list of these proteins is most probably conservative as not all proteins were 490 annotated. Nonetheless, the approach identified 151 proteins enriched for biological 491 processes associated with reproduction, mating, insemination, and sperm competition, with 492 approximately $30 \%$ that shared homology with previously identified Dmel SFPs. We also 493 compared our list with the 29 putative SFPs previously identified via genome comparison 494 between Dmel and Dpse (9). Of the nearly 50\% overlap between these two lists (14/29), 495 protein members of the canonical Sex Peptide (SP) network are particularly well-represented.

496 In Dmel, SP binds to sperm in the female seminal receptacle, a sperm storage organ, and is 497 required for both long-term female resistance to remating and for sperm release from storage 498 (62). Our list of SFPs contains several known proteins involved in the Dmel SP network, 499 including the gene duplicate pair lectins CG1652 and CG1656, aquarius (CG14061), intrepid 500 (CG12558), antares (CG30488), seminase (CG10586), CG17575 (a cysteine-rich secretory 501 protein), and CG9997 (a serine protease homolog) (16). CG9997 is processed in the female 502 and males that do not produce this protein are unable to transfer the lectins, which are required 503 to slow the rate at which CG9997 is processed in the female. All SP pathway proteins, except 504 SP, were detected in our putative list of SFPs. Absence of detectable Dpse SP protein is 505 consistent with the lack of a recognized SP ortholog in this species, and raises the interesting 506 possibility that either the Dpse SP ortholog has significantly diverged, or has been replaced 507 by another gene. If indeed a bona fide Dpse SP gene exists, further MS searches using 
508 algorithms to detect amino acid replacements (63) may be useful in the search for this elusive 509 SFP.

511 Functional compartmentalization results from divergent sexual selection

512 While our analysis found many known SFPs, $68 \%$ had no known orthology to Dmel This

513 supports the idea that SFPs and related proteins involved in reproduction show rapid evolution

514 as suggested by genome comparisons $(9,16,17,21,64-66)$ and experimental evolution

515 research on changes in sex-specific gene expression $(32,33,67)$. Here we directly measured,

516 using LC-MS/MS and LFQ, the proteome-wide effect of postcopulatory sexual selection on

517 protein abundance in two populations that had undergone over 150 generations of divergent

518 PCSS. This approach identified significant protein abundance differences in over 200 proteins

519 between the selection lines. Knowledge of the changes in protein abundance under these

520 experimental conditions provided a useful database to compare and contrast the effects of

521 selection from a proteome-wide perspective and to assess the impact of selection on the

522 protein-protein interaction networks revealed by our analysis.

524 Previous comparative work has suggested protein evolution may be adaptively 525 compartmentalized for core processes and undergoing purifying selection and for interactive 526 proteins that undergo positive selection $(24,26)$. We directly tested this assertion by 527 comparing protein interaction network architecture in the divergent PCSS treatments and 528 testing for molecular signatures of selection. We found remarkably line-specific 529 compartmentalized changes to proteins after more than 150 generations of experimental 530 sexual selection. $\mathrm{P}$ males invest in protein production machinery (i.e., protein quantity) and $\mathrm{M}$ 531 males invest in protein surveillance (i.e., protein quality). The number of matings a Drosophila 532 male can have is limited by SFP supply and how rapidly males can replenish this supply (68).

533 Moreover, males can respond plastically to increased risk of sperm competition by transferring 534 more of some SFPs to females $(14,69)$. We have previously shown that $P$ males have larger 
535 accessory glands and can mate with more females sequentially than $M$ males $(30,31)$. The

536 increased investment in protein production machinery in $\mathrm{P}$ males may underlie this phenotypic

537 response, and we suggest this may explain how males in polyandrous mating systems can

538 rapidly adjust and replenish SFP quantity. In contrast, $\mathrm{M}$ males overall have increased

539 production of proteins that function in downstream processing of secreted proteins, suggestive

540 of increased investment in protein quality. The transfer of high quality proteins to females may

541 be a response to reduce sexual conflict and improve population fitness, as predicted under

542 enforced monogamy in which the reproductive interests of the sexes become aligned.

543 The SFPs produced in larger quantities in $M$ compared to $P$ males were enriched for chitin 544 catabolic process (including wound healing) and response to fungus. Mating in Dmel initiates 545 an immune response in females that has negative fitness consequences (70). The immune 546 system may be activated to combat pathogens in the ejaculate and/or immunogenic sperm 547 and SFPs (response to fungus), and/or wounds inflicted by males (chitin catabolic processes 548 and wound healing). As enforced monogamy relaxes sexual conflict between males and 549 females, M males may produce more of these proteins to limit damage to their mates. Future 550 work could test this by manipulating these protein levels in the male ejaculate and determining 551 the consequences on female fitness following mating. $M$ males also produced more 552 ejaculatory bulb protein (Ebp; CG2668), a known constituent of the mating plug. The mating 553 plug remains in the female uterus until females eject it, along with unstored sperm. Ebp is 554 necessary for ejaculate retention; knocking down Ebp in Dmel results in ejaculate loss, and 555 reduced female sperm storage (Avila et al. 2015b). M males may produce more Ebp for better 556 ejaculate retention, reducing the likelihood of their mates requiring additional matings to 557 maintain sperm stores and subsequent fertility. Given the costs of mating for females, such a 558 response is predicted when sexual conflict is relaxed, as in the M treatment.

559 Differentially abundant P male proteins did not show significant GO BP enrichment but over $56050 \%$ of these $(5 / 9)$ are known Dmel SFPs. This includes another mating plug protein, 561 Acp53C14b, known to be involved in egg laying and reproductive fitness (71). Our previous 
562 work on the Dpse selection lines described sexual conflict over female reproductive

563 phenology; $\mathrm{M}$ females mated to $\mathrm{P}$ males oviposit more eggs earlier in their lifespan than when

564 mated to their own males (31). Given that the M treatment removes sexual conflict, alterations

565 to the $\mathrm{M}$ female oviposition schedule when mated to $\mathrm{P}$ males is likely to be costly. This

566 manipulation of female oviposition may be mediated by Acp53C14b given that this protein -

567 which $\mathrm{P}$ males produce more of - influences oviposition.

\section{Rates of molecular evolution on male reproductive proteins}

569 Rapid evolution at the molecular level is common for reproductive proteins including SFPs

570 (e.g., $(10,18,22,26))$ and this rapid evolution at the macroevolutionary scale is attributed to

571 PCSS. Here we go beyond these traditional tests and ask whether there are differences in the

572 rate of evolution between different types of reproductive proteins arising from the same tissue

573 at the microevolutionary scale following over 150 generations of experimental manipulation of

574 PCSS. We find that genes encoding SFPs showed significantly faster rates of molecular

575 evolution compared to both other secreted protein encoding genes that were not candidate

576 SFPs and overall accessory gland proteome genes. However, we also found that secretome

577 genes showed higher rates of molecular evolution than non-secreted proteins (i.e., the

578 remainder of the AcgP). These results support the interpretation that genes which interact

579 extracellularly evolve more rapidly than those that remain within the cytoplasm $(72,73)$.

580 Evolutionary rates of genes encoding differentially abundant proteins between the sexual

581 selection treatments found pronounced reduction in $\mathrm{dN}$ of $\mathrm{P}$ males compared to either

582 differentially abundant proteins in $\mathrm{M}$ or the AcgP. This was likely due to the

583 compartmentalization we identified, in which $\mathrm{P}$ males invested more in slowly evolving

584 cytosolic ribosomal proteins (Barreto and Burton 2013). However, after controlling for this, we

585 found that genes encoding for $\mathrm{M}$ - and $\mathrm{P}$ - differentially abundant proteins were more likely to

586 have been subjected to positive selection compared to the AcgP genes as a whole. This result

587 reinforces the hypothesis that the proteins we identified as responding to divergent sexual 
588 selection $\mathrm{t}$ the microevolutionary level have undergone adaptive processes on a

589 macroevolutionary scale. We argue this directly illustrates a major role of PCSS in the 590 evolution of male reproductive proteins.

\section{Conclusions}

593 By employing divergent postcopulatory sexual selection through the use of experimental 594 evolution of mating systems, we were able to directly test the hypothesis that postcopulatory sexual selection results in the rapid evolution of male-specific reproductive proteins. Within

596150 generations, substantial microevolutionary changes occurred such as differential 597 abundance of secreted proteins, including previously identified Dmel SFPs that have known 598 effects on male and female reproductive fitness. A small number of these proteins showed 599 signatures of positive molecular evolution. We found PCSS mediates remarkable 600 compartmentalization of subcellular function of the secretory tissue, suggesting sexual 601 selection alters fundamental reproductive protein networks, not just individual SFPs. These 602 changes affected protein production, but in different ways in the divergent treatments, 603 suggesting that PCSS selects for increased protein production whereas as relaxation of sexual 604 conflict selects for protein surveillance. Some specific changes may be related to previously 605 described phenotypic responses to divergent sexual selection that facilitate greater 606 reproductive capacity in males subject to polyandry and ameliorates sexual conflict in 607 populations subject to monandry. Our novel results suggest wide-spread, previously 608 unappreciated, consequences of postcopulatory sexual selection on reproductive protein 609 evolution. Combining the increasing use of high throughput proteomics and experimental 610 manipulation of mating systems in different taxa will allow broader tests of this pattern in 611 different taxa and better understanding of how sexual selection and sexual conflict impacts 612 male reproductive protein evolution.

613 Acknowledgements - We thank the many people who have contributed to the maintenance of 614 the Snook experimental evolution lines. Funding of the experimental evolution lines came from 
615 NSF (DEB-0093149), NERC (NE/B504065/1; NE/I014632/1), and EU (ITN-2008-213780

616 SPECIATION) to RRS. Funding and technical support for the proteomic work came from the

617 University of Sheffield Biological Mass Spectrophotometry Facility (funded by Yorkshire

618 Cancer Research (Shend01) and the Wellcome Trust). TIG was supported by a Leverhulme

619 Early Career Fellowship (Grant ECF-2015-453) and a NERC grant (NE/N013832/1). Funding

620 from the Kyoto Institute of Technology to TLK also supported this research.

DATA AVAILABILITY

623 Raw files for the entire datasets used in this study have been deposited via ProteomeXchange 624 on the PRIDE database repository.

\section{REFERENCES}

628 1. Snook, R. R. (2014) The evolution of polyandry. The Evolution of Insect Mating 629 Systems 159-180

630 2. Arnqvist, G. and Rowe, L. (2005) Sexual conflict, Princeton University Press, 631 Princeton, N.J.

632 3. Birkhead, T. R., Hosken, D. J. and Pitnick, S. S. (2008) Sperm Biology.

633 4. Avila, F. W., Sirot, L. K., LaFlamme, B. A., Rubinstein, C. D. and Wolfner, M. F. (2011)

634 Insect seminal fluid proteins: identification and function. Annual review of entomology 56, 2163540

636 5. Ramm, S. A., McDonald, L., Hurst, J. L., Beynon, R. J. and Stockley, P. (2009)

637 Comparative proteomics reveals evidence for evolutionary diversification of rodent seminal

638 fluid and its functional significance in sperm competition. Mol Biol Evol 26, 189-198

639 6. Dorus, S., Evans, P. D., Wyckoff, G. J., Choi, S. S. and Lahn, B. T. (2004) Rate of 640 molecular evolution of the seminal protein gene SEMG2 correlates with levels of female 641 promiscuity. Nat Genet 36, 1326-1329

642 7. Harrison, P. W., Wright, A. E., Zimmer, F., Dean, R., Montgomery, S. H., Pointer, M. 
643 A. and Mank, J. E. (2015) Sexual selection drives evolution and rapid turnover of male gene 644 expression. Proc Natl Acad Sci U S A 112, 4393-4398

645 8. Panhuis, T. M., Clark, N. L. and Swanson, W. J. (2006) Rapid evolution of reproductive 646 proteins in abalone and Drosophila. Philosophical transactions of the Royal Society of London 647 B: Biological sciences 361, 261-268

648 9. Mueller, J. L., Ravi Ram, K., McGraw, L. A., Bloch Qazi, M. C., Siggia, E. D., Clark, A. 649 G., Aquadro, C. F. and Wolfner, M. F. (2005) Cross-species comparison of Drosophila male 650 accessory gland protein genes. Genetics 171, 131-143

651 10. Clark, N. L., Aagaard, J. E. and Swanson, W. J. (2006) Evolution of reproductive 652 proteins from animals and plants. Reproduction 131, 11-22

653 11. Mattei, A. L., Riccio, M. L., Avila, F. W. and Wolfner, M. F. (2015) Integrated 3D view 654 of postmating responses by the Drosophila melanogaster female reproductive tract, obtained 655 by micro-computed tomography scanning. Proceedings of the National Academy of Sciences of the United States of America 112, 8475-8480

657 12. Avila, F. W. and Wolfner, M. F. (2009) Acp36DE is required for uterine conformational 658 changes in mated Drosophila females. Proceedings of the National Academy of Sciences of 659 the United States of America 106, 15796-15800

660 13. Perry, J. C., Sirot, L. and Wigby, S. (2013) The seminal symphony: How to compose 661 an ejaculate. Trends in Ecology and Evolution 28, 414-422

662 14. Wigby, S., Sirot, L. K., Linklater, J. R., Buehner, N., Calboli, F. C. F., Bretman, A., 663 Wolfner, M. F. and Chapman, T. (2009) Seminal fluid protein allocation and male reproductive 664 success. Current biology : CB 19, 751-757

665 15. Avila, F. W., Mattei, A. L. and Wolfner, M. F. (2015) Sex peptide receptor is required 666 for the release of stored sperm by mated Drosophila melanogaster females. Journal of insect 667 physiology $76,1-6$

668 16. Findlay, G. D., Sitnik, J. L., Wang, W., Aquadro, C. F., Clark, N. L. and Wolfner, M. F. 669 (2014) Evolutionary rate covariation identifies new members of a protein network required for 670 Drosophila melanogaster female post-mating responses. PLoS Genetics 10, e1004108 
671 17. Swanson, W. J., Clark, A. G., Waldrip-Dail, H. M., Wolfner, M. F. and Aquadro, C. F.

672 (2001) Evolutionary EST analysis identifies rapidly evolving male reproductive proteins in

673 Drosophila. Proceedings of the National Academy of Sciences of the United States of America

$67498,7375-7379$

675 18. Mueller, J. L., Ripoll, D. R., Aquadro, C. F. and Wolfner, M. F. (2004) Comparative

676 structural modeling and inference of conserved protein classes in Drosophila seminal fluid.

677 Proceedings of the National Academy of Sciences of the United States of America 101, 13542-

$678 \quad 13547$

679 19. Wagstaff, B. J. and Begun, D. J. (2005) Comparative genomics of accessory gland 680 protein genes in Drosophila melanogaster and D. pseudoobscura. Mol Biol Evol 22, 818-832

681 20. Findlay, G. D., MacCoss, M. J. and Swanson, W. J. (2009) Proteomic discovery of 682 previously unannotated, rapidly evolving seminal fluid genes in Drosophila. Genome research $68319,886-896$

684 21. Findlay, G. D., Yi, X., Maccoss, M. J. and Swanson, W. J. (2008) Proteomics reveals 685 novel Drosophila seminal fluid proteins transferred at mating. PLoS Biol 6, e178

686 22. Wilburn, D. B. and Swanson, W. J. (2016) From molecules to mating: Rapid evolution 687 and biochemical studies of reproductive proteins. J Proteomics 135, $12-25$

688 23. Schumacher, J., Rosenkranz, D. and Herlyn, H. (2014) Mating systems and protein689 protein interactions determine evolutionary rates of primate sperm proteins. Proc Biol Sci 281, $690 \quad 20132607$

691 24. Dorus, S., Wasbrough, E. R., Busby, J., Wilkin, E. C. and Karr, T. L. (2010) Sperm 692 proteomics reveals intensified selection on mouse sperm membrane and acrosome genes. 693 Molecular biology and evolution 27, 1235-1246

694 25. Dorus, S., Busby, S. A., Gerike, U., Shabanowitz, J., Hunt, D. F. and Karr, T. L. (2006) 695 Genomic and functional evolution of the Drosophila melanogaster sperm proteome. Nature 696 genetics $38,1440-1445$

697 26. Vicens, A., Borziak, K., Karr, T. L., Roldan, E. R. S. and Dorus, S. (2017) Comparative 698 Sperm Proteomics in Mouse Species with Divergent Mating Systems. Mol Biol Evol 34, 1403- 
700 27. Athanasios, A., Charalampos, V., Vasileios, T. and Ashraf, G. M. (2017) Protein-

701 Protein Interaction (PPI) Network: Recent Advances in Drug Discovery. Curr Drug Metab 18, $702 \quad 5-10$

703 28. Levy, E. D. and Pereira-Leal, J. B. (2008) Evolution and dynamics of protein 704 interactions and networks. Curr Opin Struct Biol 18, 349-357

705 29. Crudgington, H. S., Beckerman, A. P., Brustle, L., Green, K. and Snook, R. R. (2005)

706 Experimental removal and elevation of sexual selection: Does sexual selection generate 707 manipulative males and resistant females? American Naturalist 165, S72-S87

708 30. Crudgington, H. S., Fellows, S. and Snook, R. R. (2010) Increased opportunity for 709 sexual conflict promotes harmful males with elevated courtship frequencies. Journal of 710 evolutionary biology $23,440-446$

711 31. Crudgington, H. S., Fellows, S., Badcock, N. S. and Snook, R. R. (2009) Experimental 712 manipulation of sexual selection promotes greater male mating capacity but does not alter 713 sperm investment. Evolution; international journal of organic evolution 63, 926-938

714 32. Immonen, E., Snook, R. R. and Ritchie, M. G. (2014) Mating system variation drives 715 rapid evolution of the female transcriptome in Drosophila pseudoobscura. Ecology and 716 evolution $4,2186-2201$

717 33. Veltsos, P., Fang, Y., Cossins, A. R., Snook, R. R. and Ritchie, M. G. (2017) Mating 718 system manipulation and the evolution of sex-biased gene expression in Drosophila. Nat 719 Commun 8, 2072

720 34. Anderson, W. W. (1974) Frequent multiple insemination in a natural population of 721 Drosophila pseudoobscura. The American naturalist 108, 709-711

722 35. Snook, R. R., Brüstle, L. and Slate, J. (2009) A test and review of the role of effective 723 population size on experimental sexual selection patterns. Evolution; international journal of 724 organic evolution $63,1923-1933$

725 36. Snook, R. R. and Markow, T. A. (2002) Efficiency of gamete usage in nature: sperm 726 storage, fertilization and polyspermy. Proceedings. Biological sciences / The Royal Society 
727

728

729

730

731

732

733

734

735

736

737

738

739

740

741

742

743

744

745

746

747

748

749

750

751

752

753

754

269, 467-473

37. Nesvizhskii, A. I., Keller, A., Kolker, E. and Aebersold, R. (2003) A Statistical Model for Identifying Proteins by Tandem Mass Spectrometry abilities that proteins are present in a sample on the basis. Analytical chemistry 75, 4646-4658

38. Kriventseva, E. V., Tegenfeldt, F., Petty, T. J., Waterhouse, R. M., Simão, F. A., Pozdnyakov, I. A., Ioannidis, P. and Zdobnov, E. M. (2015) OrthoDB v8: update of the hierarchical catalog of orthologs and the underlying free software. Nucleic Acids Res 43, D250-6

39. Petersen, T. N., Brunak, S., von Heijne, G. and Nielsen, H. (2011) SignalP 4.0: discriminating signal peptides from transmembrane regions. Nature methods $\mathbf{8}, \mathbf{7 8 5 - 7 8 6}$

40. Käll, L., Krogh, A. and Sonnhammer, E. L. (2004) A combined transmembrane topology and signal peptide prediction method. J Mol Biol 338, 1027-1036

41. Cline, M. S., Smoot, M., Cerami, E., Kuchinsky, A., Landys, N., Workman, C., Christmas, R., Avila-Campilo, I., Creech, M., Gross, B., Hanspers, K., Isserlin, R., Kelley, R., Killcoyne, S., Lotia, S., Maere, S., Morris, J., Ono, K., Pavlovic, V., Pico, A. R., Vailaya, A., Wang, P.-L., Adler, A., Conklin, B. R., Hood, L., Kuiper, M., Sander, C., Schmulevich, I., Schwikowski, B., Warner, G. J., Ideker, T. and Bader, G. D. (2007) Integration of biological networks and gene expression data using Cytoscape. Nature protocols 2, 2366-2382

42. Demchak, B., Hull, T., Reich, M., Liefeld, T., Smoot, M., Ideker, T. and Mesirov, J. P. (2014) Cytoscape: the network visualization tool for GenomeSpace workflows.

\section{F1000Research 3, 151}

43. Bindea, G., Mlecnik, B., Hackl, H., Charoentong, P., Tosolini, M., Kirilovsky, A., Fridman, W.-H., Pagès, F., Trajanoski, Z. and Galon, J. (2009) ClueGO: A Cytoscape plug-in to decipher functionally grouped gene ontology and pathway annotation networks. Bioinformatics (Oxford, England) 25, 1091-1093

44. Bindea, G., Galon, J. and Mlecnik, B. (2013) CluePedia Cytoscape plugin: pathway insights using integrated experimental and in silico data. Bioinformatics 29, 661-663

$$
\text { 45. Szklarczyk, D., Franceschini, A., Wyder, S., Forslund, K., Heller, D., Huerta-Cepas, J., }
$$


755 Simonovic, M., Roth, A., Santos, A., Tsafou, K. P., Kuhn, M., Bork, P., Jensen, L. J. and von

756 Mering, C. (2015) STRING v10: protein-protein interaction networks, integrated over the tree

757 of life. Nucleic Acids Res 43, D447-52

758 46. Choi, H., Fermin, D. and Nesvizhskii, A. I. (2008) Significance Analysis of Spectral

759 Count Data in Label-free Shotgun Proteomics. Molecular \& Cellular Proteomics 7, 2373-2385

760 47. Choi, H., Kim, S., Fermin, D., Tsou, C. C. and Nesvizhskii, A. I. (2015) QPROT:

761 Statistical method for testing differential expression using protein-level intensity data in label-

762 free quantitative proteomics. J Proteomics 129, 121-126

763 48. Blein-Nicolas, M. and Zivy, M. (2016) Thousand and one ways to quantify and compare 764 protein abundances in label-free bottom-up proteomics. Biochimica et biophysica acta 1864, $765 \quad 883-895$

766 49. Palmieri, N., Kosiol, C. and Schlötterer, C. (2014) The life cycle of Drosophila orphan 767 genes. Elife 3, e01311

768 50. Altschul, S. F., Gish, W., Miller, W., Myers, E. W. and Lipman, D. J. (1990) Basic local 769 alignment search tool. J Mol Biol 215, 403-410

770 51. Gossmann, T. I., Santure, A. W., Sheldon, B. C., Slate, J. and Zeng, K. (2014) Highly 771 variable recombinational landscape modulates efficacy of natural selection in birds. Genome 772 Biol Evol 6, 2061-2075

773 52. Edgar, R. C. (2004) MUSCLE: multiple sequence alignment with high accuracy and 774 high throughput. Nucleic Acids Res 32, 1792-1797

775 53. Wu, M., Chatterji, S. and Eisen, J. A. (2012) Accounting for alignment uncertainty in 776 phylogenomics. PLoS One 7, e30288

777 54. Suyama, M., Torrents, D. and Bork, P. (2006) PAL2NAL: robust conversion of protein 778 sequence alignments into the corresponding codon alignments. Nucleic Acids Res 34, W609$779 \quad 12$

780 55. Yang, Z. (2007) PAML 4: phylogenetic analysis by maximum likelihood. Mol Biol Evol

$781 \quad 24,1586-1591$

782 56. Hochberg, Y. and Benjamini, Y. (1990) More powerful procedures for multiple 
784 57. Mann, H. B. and Whitney, D. R. (1947) On a Test of Whether One of Two Random

785 Variables Is Stochastically Larger Than the Other. Ann. Math. Statistics 18, 50-60

786 58. Armengaud, J., Christie-Oleza, J. A., Clair, G., Malard, V. and Duport, C. (2012)

787 Exoproteomics: exploring the world around biological systems. Expert Rev Proteomics 9, 561-

$788 \quad 575$

789 59. Tjalsma, H., Bolhuis, A., Jongbloed, J. D., Bron, S. and van Dijl, J. M. (2000) Signal

790 peptide-dependent protein transport in Bacillus subtilis: a genome-based survey of the 791 secretome. Microbiology and molecular biology reviews : MMBR 64, 515-547

792 60. Käll, L., Krogh, A. and Sonnhammer, E. L. (2007) Advantages of combined 793 transmembrane topology and signal peptide prediction--the Phobius web server. Nucleic

794 Acids Res 35, W429-32

795 61. Barreto, F. S. and Burton, R. S. (2013) Evidence for compensatory evolution of 796 ribosomal proteins in response to rapid divergence of mitochondrial rRNA. Mol Biol Evol 30, $797 \quad 310-314$

798 62. Avila, F. W., Ravi Ram, K., Bloch Qazi, M. C. and Wolfner, M. F. (2010) Sex peptide 799 is required for the efficient release of stored sperm in mated Drosophila females. Genetics $800186,595-600$

801 63. Starkweather, R., Barnes, C. S., Wyckoff, G. J. and Keightley, J. A. (2007) Virtual 802 polymorphism: finding divergent peptide matches in mass spectrometry data. Anal Chem 79, $803 \quad 5030-5039$

804 64. Clark, N. L. and Swanson, W. J. (2005) Pervasive adaptive evolution in primate 805 seminal proteins. PLoS Genet 1, e35

806 65. Dean, M. D., Clark, N. L., Findlay, G. D., Karn, R. C., Yi, X., Swanson, W. J., MacCoss, 807 M. J. and Nachman, M. W. (2009) Proteomics and comparative genomic investigations reveal 808 heterogeneity in evolutionary rate of male reproductive proteins in mice (Mus domesticus). $809 \mathrm{Mol}$ Biol Evol 26, 1733-1743

810 66. Sirot, L. K., Findlay, G. D., Sitnik, J. L., Frasheri, D., Avila, F. W. and Wolfner, M. F. 
811 (2014) Molecular characterization and evolution of a gene family encoding both female- and

812 male-specific reproductive proteins in Drosophila. Molecular biology and evolution 31, 1554-

$813 \quad 1567$

814 67. Hollis, B., Houle, D., Yan, Z., Kawecki, T. J. and Keller, L. (2014) Evolution under

815 monogamy feminizes gene expression in Drosophila melanogaster. Nat Commun 5, 3482

816 68. Linklater, J. R., Wertheim, B., Wigby, S. and Chapman, T. (2007) Ejaculate depletion

817 patterns evolve in response to experimental manipulation of sex ratio in Drosophila

818 melanogaster. Evolution 61, 2027-2034

819 69. Sirot, L. K., Wolfner, M. F. and Wigby, S. (2011) Protein-specific manipulation of

820 ejaculate composition in response to female mating status in Drosophila melanogaster.

821 Proceedings of the National Academy of Sciences of the United States of America 108, 9922-

8229926

823 70. Mack, P. D., Kapelnikov, A., Heifetz, Y. and Bender, M. (2006) Mating-responsive

824 genes in reproductive tissues of female Drosophila melanogaster. Proc Natl Acad Sci U S A

$825103,10358-10363$

826 71. Avila, F. W., Cohen, A. B., Ameerudeen, F. S., Duneau, D., Suresh, S., Mattei, A. L.

827 and Wolfner, M. F. (2015) Retention of Ejaculate by Drosophila melanogaster Females

828 Requires the Male-Derived Mating Plug Protein PEBme. Genetics 200, 1171-1179

829 72. Feyertag, F., Berninsone, P. M. and Alvarez-Ponce, D. (2017) Secreted Proteins Defy

830 the Expression Level-Evolutionary Rate Anticorrelation. Mol Biol Evol 34, 692-706

831 73. Liao, B. Y., Weng, M. P. and Zhang, J. (2010) Impact of extracellularity on the

832 evolutionary rate of mammalian proteins. Genome Biol Evol 2, 39-43

833

834 
835 Figure Legends

836

837 Figure 1. Dpse accessory gland summary statistics. (A) Venn diagram of combined and total

838 Uniprot IDs from the M- and P-experimental lines used to assemble the AcgP. (B) Secretome 839 and exoproteome from the AcgP derived in silico (see Methods). (C) Differential protein 840 abundance between the $\mathrm{M}$ - and $\mathrm{P}$ - experimental lines. Numbers in parentheses indicate Dmel 841 orthologs from OrthoDB database.

843 Figure 2. AcgP GO category enrichment analysis. Distribution of major functional category 844 groupings for Biological Process (A) and Cellular Component (B). Categories related to 845 secretory processes and discussed in main text highlighted in bold.

847 Figure 3. Functional grouping and subcellular localizaton of the AcgS mapped to subcellular 848 regions (labeled on the right) using the Cerebral layout in Cytoscape. Shaded regions show 849 all annotations that include the keyword "Extracellular".

851 Figure 4. Network and predicted cellular distribution of DE proteins. (A) Overview of the DE 852 proteins (M- and P-lines combined) showing major enriched functional groupings for GO 853 Cellular Components (CC) using Cytoscape and Cluego. The four major groups, cytoskeletal 854 part, golgi part, translation machinery and endomembrane system are shaded with different 855 background colors. Dashed line encloses two functionally distinct categories, the ribosome 856 and tRNA synthetase complex of the translation machinery (see also Table 5). (B) Network 857 comparison of functional annotation groupings between DE proteins of the $\mathrm{M}$ - and P-lines. 858 DE proteins were segregated into their respective $\mathrm{M}$ - or $\mathrm{P}$ - lines based on relative protein 859 abundance and both groups compared using Cluego. A heat map (red-to-green) shows the 860 distribution of DE protein between the $\mathrm{M}$ - and $\mathrm{P}$-lines. These distributions were then mapped 861 onto the predicted cellular locations of the groupings using the Cluepedia and the Cerebral 862 layout as implemented in Cytoscape. 
864 Figure 5. PPI network representation of the $M($ red, $M>P$ ) and $P$ (green, $P>M)$ DE proteins

865 (inset). The PPI network was generated using STRING, a program that determines protein-

866 protein interactions based on experimental and database criteria and calculated confidence

867 levels to assign interactions between protein pairs. Stippled lines indicate major functional

868 categories describing the networks.

869

870 Figure 6. (A) Evolutionary rate calculations of the ratio of the non-synonymous to synonymous

871 (dN/dS) substitution rates for (left to right) the Acg proteome (AgcP), the secretome (AcgS,

872 excluding exoproteome and the exoproteome (AcgS-Extracellular). (B) The rate of

873 nonsynonynous changes ( $\mathrm{dn}$ ) in genes coding for (left to right); the AcgP (minus DE M and P

874 treatment genes), differentially abundant M-line genes (DE_M_UP) and differentially abundant

875 P-line genes (DE_P_UP). Number of genes analyzed in each category listed below category.

876 (C) Summary table of evolutionary rate analysis indicating significant enrichment of differential 877 abundance proteins under positive selection. 

not certified by peer review) is the author/funder. All rights reserved. No reuse allowed without permission.

Table 1. Enriched KEGG pathways of the AcgP.

\begin{tabular}{clcc} 
GOID & \multicolumn{1}{c}{ GOTerm } & Nr. Genes & Pvalue $^{*}$ \\
\hline KEGG:03008 & Ribosome biogenesis & 33 & $3.5 \mathrm{E}-12$ \\
KEGG:03040 & Spliceosome & 82 & $8.0 \mathrm{E}-06$ \\
KEGG:04141 & Processing in endoplasmic reticulum & 82 & $5.9 \mathrm{E}-05$ \\
KEGG:03018 & RNA degradation & 40 & $4.6 \mathrm{E}-04$ \\
KEGG:03050 & Proteasome & 35 & $5.5 \mathrm{E}-04$ \\
KEGG:04144 & Endocytosis & 74 & $5.6 \mathrm{E}-04$ \\
KEGG:04310 & Wnt signaling pathway & 24 & $1.8 \mathrm{E}-03$ \\
KEGG:00071 & Fatty acid degradation & 24 & $2.9 \mathrm{E}-03$ \\
KEGG:04130 & SNARE vesicular transport & 17 & $2.9 \mathrm{E}-03$ \\
KEGG:00330 & Arginine and proline metabolism & 34 & $4.6 \mathrm{E}-03$ \\
KEGG:00230 & Purine metabolism & 40 & $1.0 \mathrm{E}-02$ \\
KEGG:03060 & Protein export & 17 & $1.1 \mathrm{E}-02$ \\
KEGG:00280 & Valine, leucine and isoleucine degradation & 22 & $1.3 \mathrm{E}-02$ \\
KEGG:00250 & Ala, asp and glu metabolism & 20 & $1.4 \mathrm{E}-02$ \\
KEGG:00020 & Citrate cycle (TCA cycle) & 27 & $2.5 \mathrm{E}-02$ \\
KEGG:00030 & Pentose phosphate pathway & 16 & $2.8 \mathrm{E}-02$ \\
KEGG:04068 & FoxO signaling pathway & 16 & $2.9 \mathrm{E}-02$ \\
KEGG:00500 & Starch and sucrose metabolism & 20 & $4.2 \mathrm{E}-02$ \\
KEGG:03010 & Ribosome & 83 & $4.3 \mathrm{E}-02$ \\
KEGG:03015 & mRNA surveillance pathway & 41 & $4.4 \mathrm{E}-02$ \\
\hline
\end{tabular}

*Bonferroni Corrected 
Table 2. Enriched BP categories of the AcgS exoproteome (putative SFPs).

\begin{tabular}{|c|c|c|}
\hline BP Category & Nr. Genes & Pvalue* \\
\hline multicellular organism reproduction & 77 & $7.5 \mathrm{E}-29$ \\
\hline insemination & 11 & $2.8 \mathrm{E}-13$ \\
\hline drug catabolic process & 10 & 1.3E-06 \\
\hline response to biotic stimulus & 20 & 7.3E-06 \\
\hline lipid transport & 9 & 2.2E-05 \\
\hline response to wounding & 8 & $1.9 \mathrm{E}-03$ \\
\hline negative regulation of catalytic activity & 9 & $2.4 \mathrm{E}-03$ \\
\hline reproduction & 78 & 3.3E-27 \\
\hline multi-multicellular organism process & 11 & $2.8 \mathrm{E}-13$ \\
\hline copulation & 11 & 2.7E-12 \\
\hline sperm competition & 10 & $5.6 \mathrm{E}-12$ \\
\hline regulation of female receptivity, post-mating & 9 & $2.0 \mathrm{E}-11$ \\
\hline regulation of female receptivity & 9 & 1.7E-09 \\
\hline female mating behavior & 9 & $2.0 \mathrm{E}-08$ \\
\hline response to external biotic stimulus & 20 & 7.3E-06 \\
\hline response to other organism & 20 & 7.3E-06 \\
\hline carbohydrate derivative catabolic process & 9 & $1.1 \mathrm{E}-05$ \\
\hline mating & 12 & 4.5E-05 \\
\hline reproductive behavior & 12 & $6.2 \mathrm{E}-05$ \\
\hline mating behavior & 11 & $9.4 \mathrm{E}-05$ \\
\hline response to bacterium & 13 & 5.5E-04 \\
\hline lipid localization & 9 & 9.6E-04 \\
\hline aminoglycan metabolic process & 8 & $2.5 \mathrm{E}-03$ \\
\hline negative regulation of molecular function & 9 & $3.5 \mathrm{E}-03$ \\
\hline
\end{tabular}

*Bonferonni corrected 
Table 3

19 genes with site-specific test of positive selection (PAML; M7a vs M8a)

\begin{tabular}{lllcc}
\hline Dpse gene & Dpse FBgn ID & Dmel FBgn ID & Symbol name & Diff Abundance† \\
\hline GA20754 & FBgn0080745 & FBgn0262559 & Mdh2 & M-Up \\
GA16372 & FBgn0076387 & FBgn0051659 & CG31659 & M-Up \\
GA13959 & FBgn0073991 & FBgn0265434 & zip & P-Up \\
GA18802 & FBgn0078802 & FBgn0001098 & Gdh & P-Up \\
GA19458 & FBgn0079454 & FBgn0027582 & CG6230 & P-Up \\
GA23027 & FBgn0244429 & FBgn0264078 & Flo2 & \\
GA16248 & FBgn0076264 & FBgn0038197 & foxo & \\
GA14679 & FBgn0074706 & FBgn0027872 & rdgBbeta & \\
GA10763 & FBgn0070819 & FBgn0033160 & Dhx15 & \\
GA11564 & FBgn0071615 & FBgn0037489 & CG1234 & \\
GA19689 & FBgn0079685 & FBgn0037842 & CG6567 & \\
GA20526 & FBgn0080520 & FBgn0261065 & Cpsf73 & \\
GA20026 & FBgn0080022 & FBgn0035121 & Tudor-SN & \\
GA21237 & FBgn0081225 & FBgn0024238 & Fim & \\
GA17987 & FBgn0077996 & FBgn0000299 & Col4a1 & \\
GA14182 & FBgn0074211 & FBgn0016075 & vkg & \\
GA13434 & FBgn0073470 & FBgn0035533 & Cip4 & \\
GA16683 & FBgn0076698 & FBgn0052104 & CG32104 & \\
GA25309 & FBgn0246690 & ND* & ND & \\
\hline
\end{tabular}

* no ortholog found

tDA proteins (bold) from the indicated selection lines ( $M$ or $P$ ) with evidence of positive selection 
A

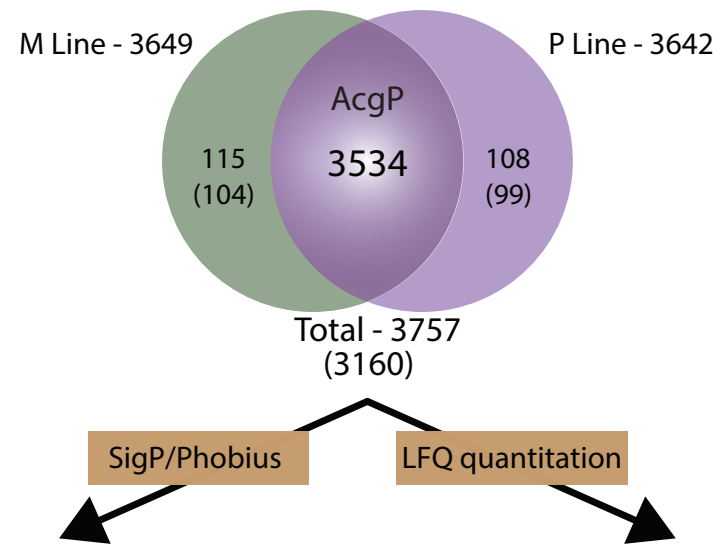

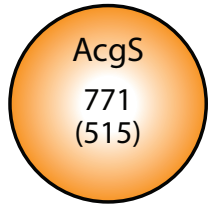

Secretome

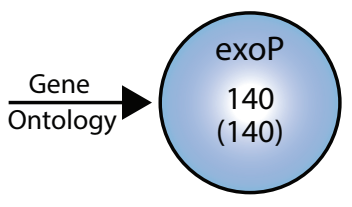

Exoproteome

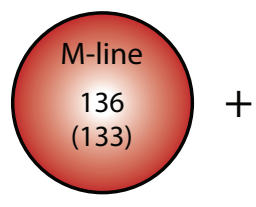

P-line

103

(96)

Differential abundance (239) 
bioRxiv preprint doi: https://doi.org/10.1101/422410; this version posted September 21, 2018. The copyright holder for this preprint (which was not certified by peer review) is the author/funder. All rights reserved. No reuse allowed without permission.

Figure 2

\section{GO Catergory Enrichment (\% terms per group)}

A
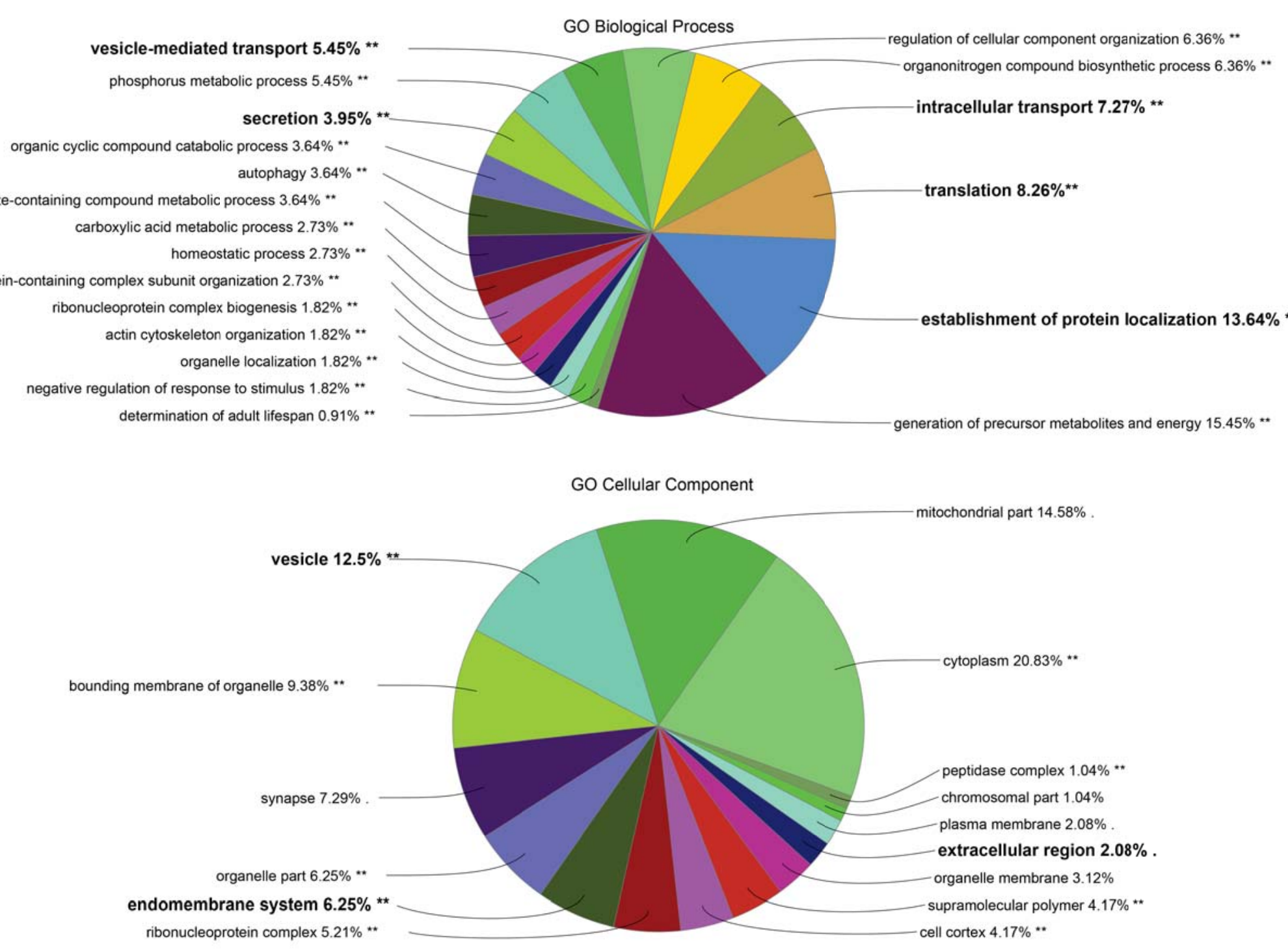
Figure 3

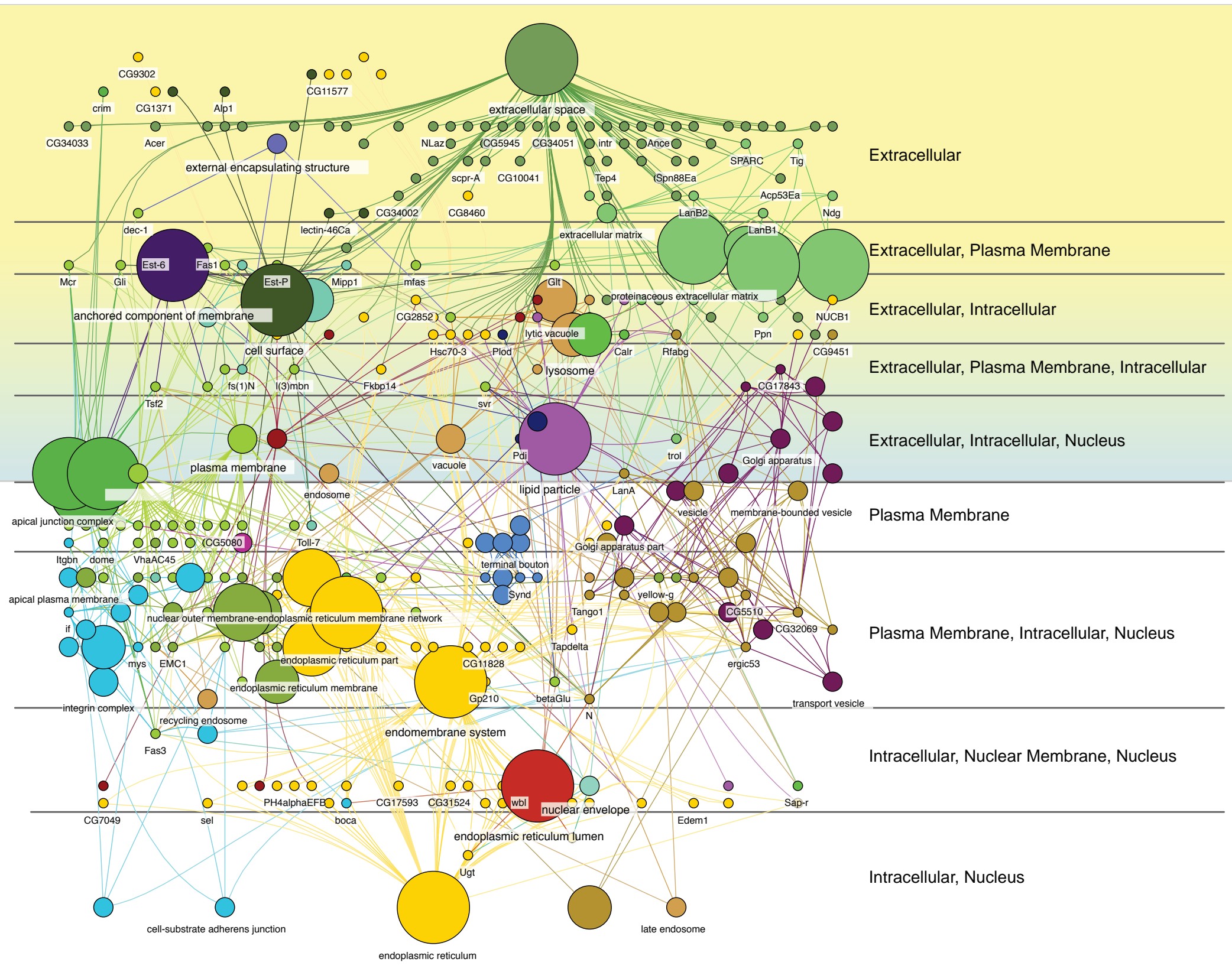


bioRxiv preprint doi:Attps://doi.org/10.11Q1/422410; this version posted September 21, 2018. The copyright holder for this preprint (which was not certified by peer reviev) is the author/funder. All rightippies

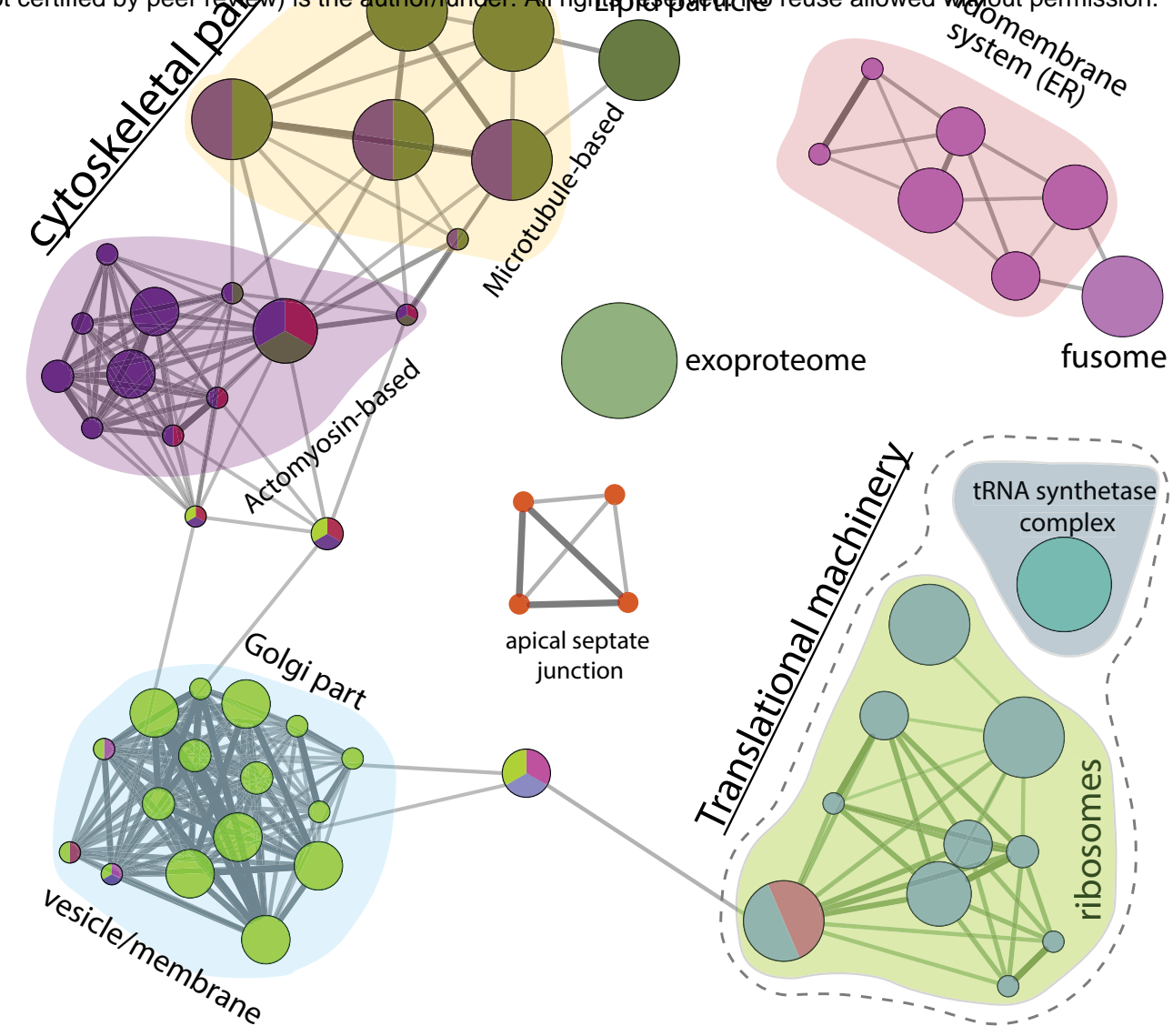

Figure 4

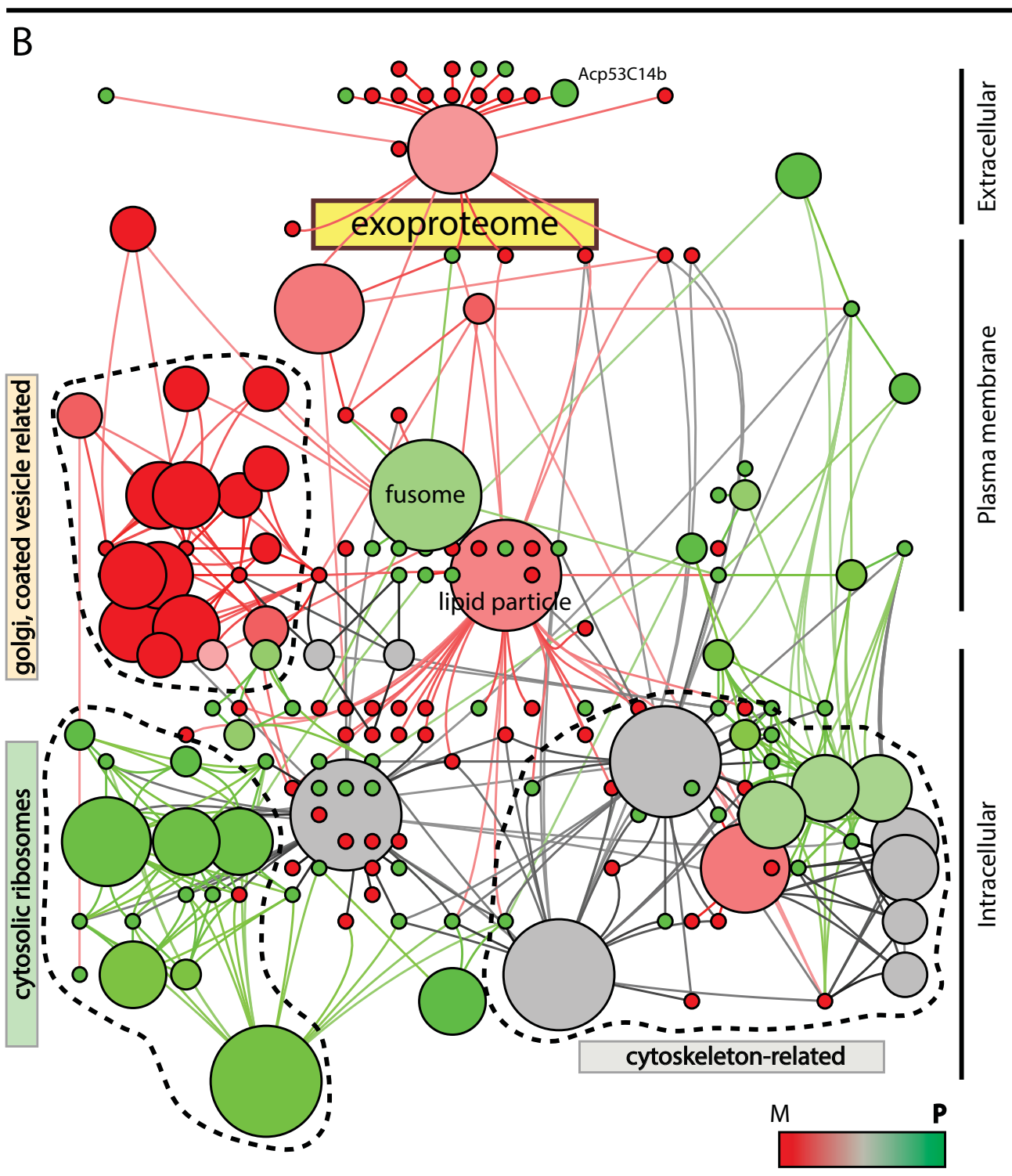


Figure 5
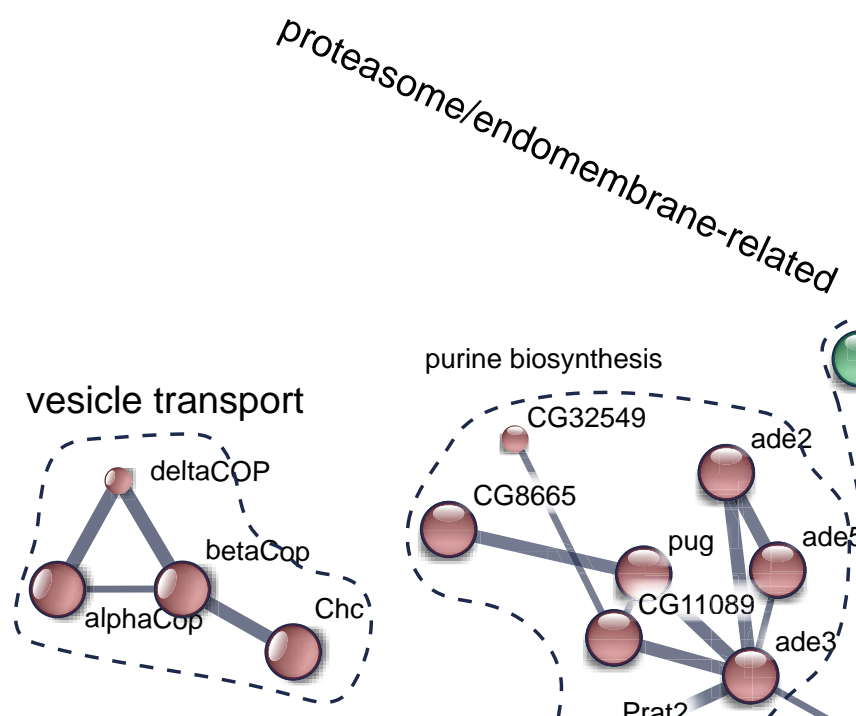

$$
\text { purine biosynthesis }
$$
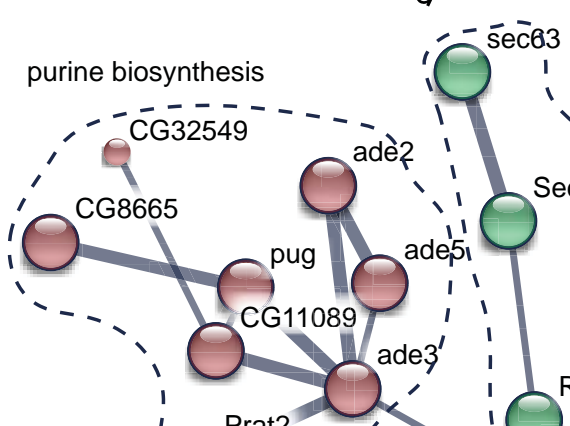

fatty acid/lipid biosynthesis and regulation

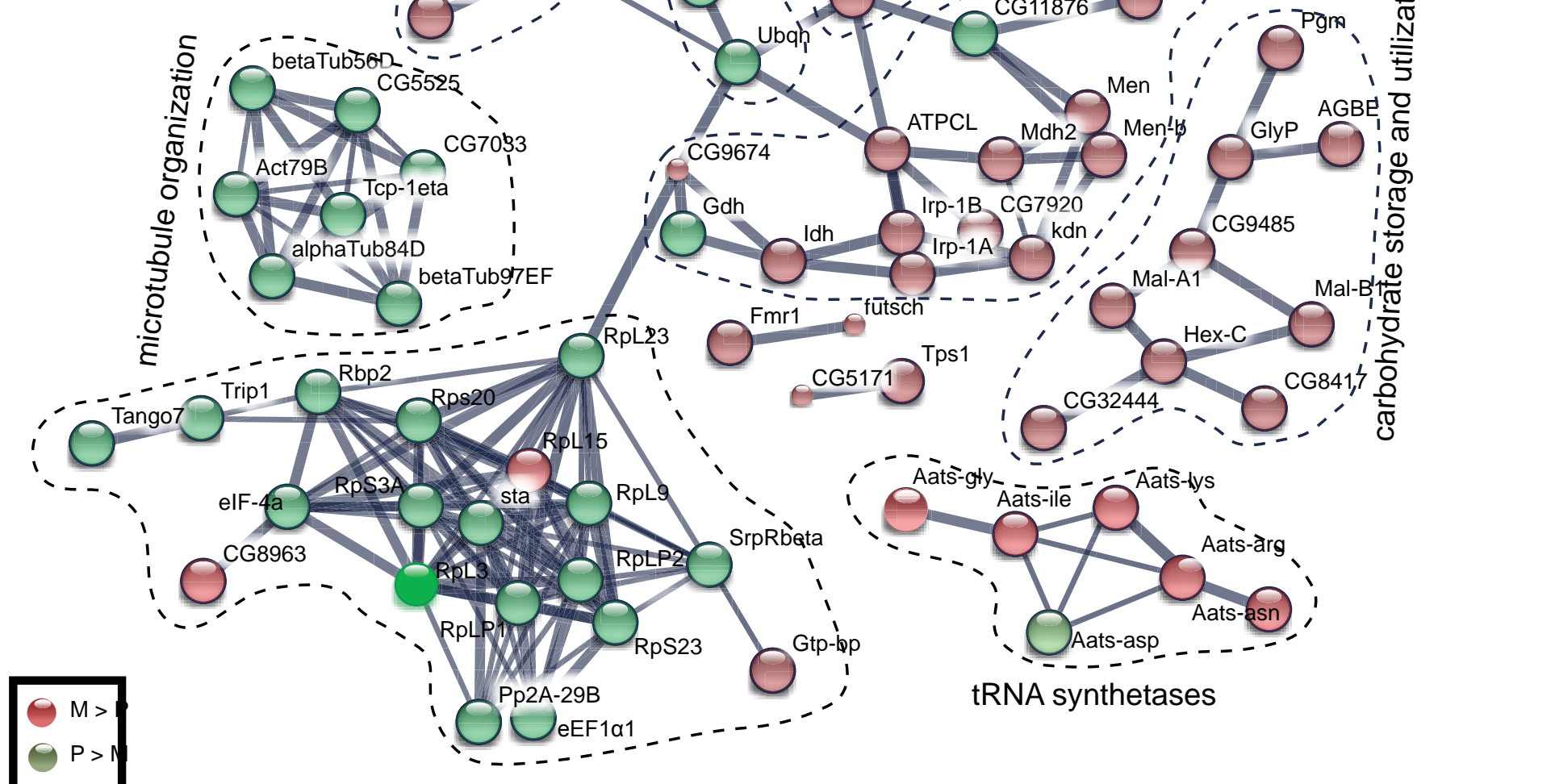

Ribosomes 

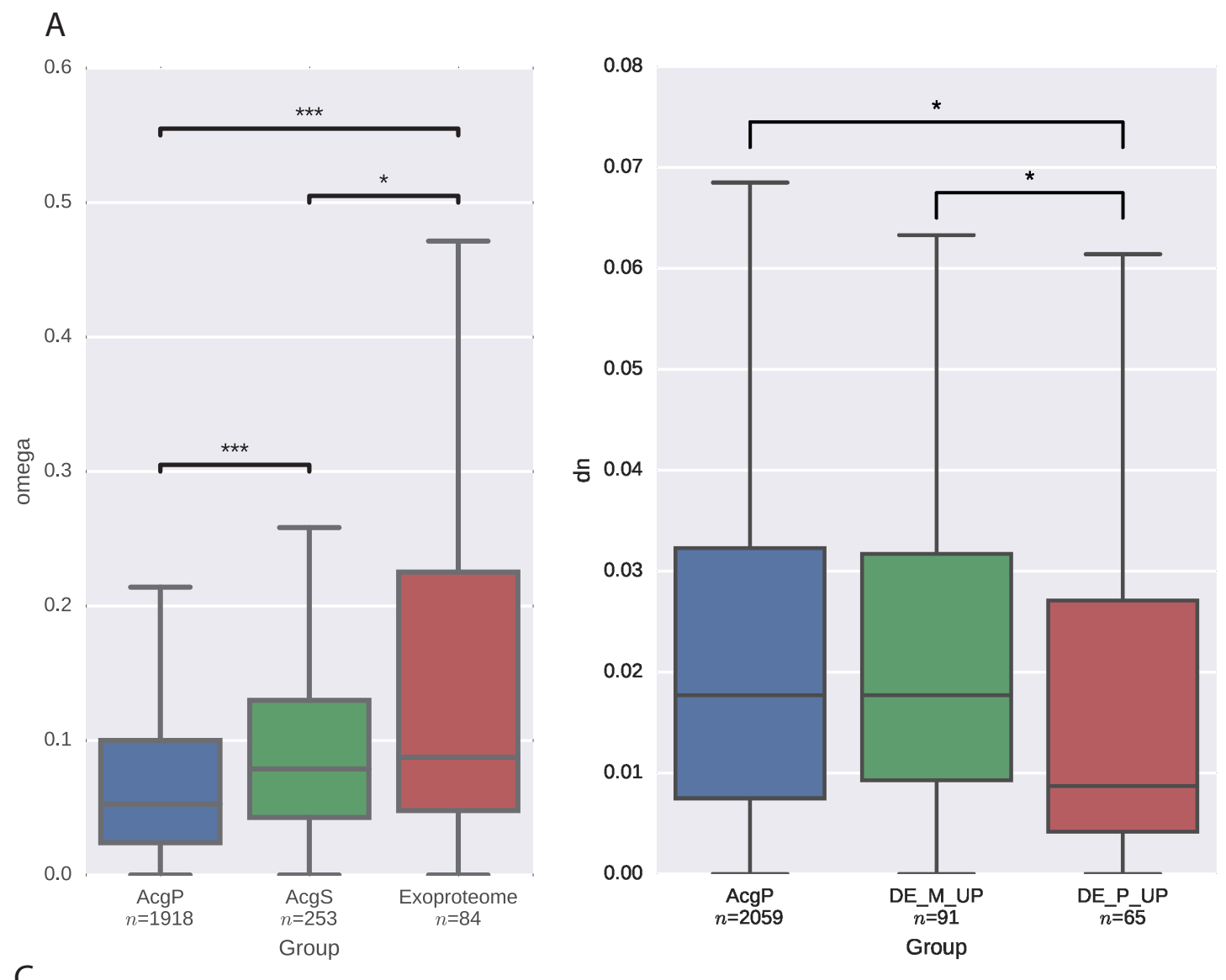

C

Evolutionary Rate Analysis of Accessory Gland Proteome

\begin{tabular}{cccc}
\hline Protein Group & (+)ive selection & no (+) selection & P-value $^{*}$ \\
\hline AcgP & 14 & 2045 & NA \\
\hline P-line & 3 & 62 & 0.0137 \\
\hline M-line & 2 & 89 & 0.1449 \\
\hline M+P-line & 5 & 151 & 0.0084 \\
\hline
\end{tabular}

*2x2 Fisher's exact test (vs AcgP) 